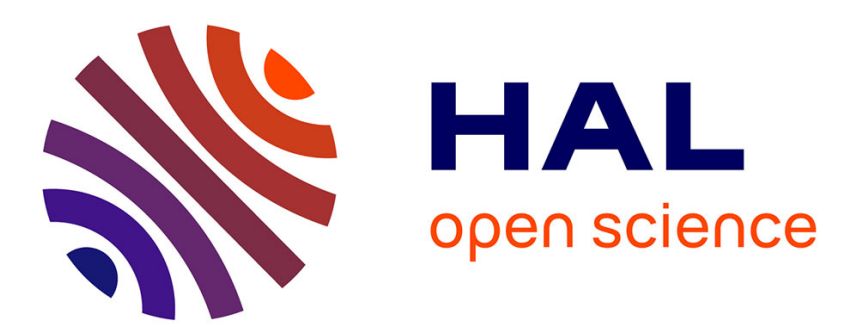

\title{
Propriétés diélectriques de l'hexaméthylène adipamide a humidité contrôlée, en très basse fréquence (103-10-4 $\mathrm{Hz}$ )
}

R. Michel, M. Maitrot, G. Asch

\section{- To cite this version:}

R. Michel, M. Maitrot, G. Asch. Propriétés diélectriques de l'hexaméthylène adipamide a humidité contrôlée, en très basse fréquence (103-10-4 Hz). Revue de Physique Appliquée, 1973, 8 (4), pp.315323. 10.1051/rphysap:0197300804031500 . jpa-00243689

\section{HAL Id: jpa-00243689 https://hal.science/jpa-00243689}

Submitted on 1 Jan 1973

HAL is a multi-disciplinary open access archive for the deposit and dissemination of scientific research documents, whether they are published or not. The documents may come from teaching and research institutions in France or abroad, or from public or private research centers.
L'archive ouverte pluridisciplinaire HAL, est destinée au dépôt et à la diffusion de documents scientifiques de niveau recherche, publiés ou non, émanant des établissements d'enseignement et de recherche français ou étrangers, des laboratoires publics ou privés. 


\title{
PROPRIÉTÉS DIÉLECTRIQUES DE L'HEXAMÉTHYLẼNE ADIPAMIDE A HUMIDITÉ CONTRÔLÉE, EN TRÈS BASSE FRÉQUENCE $\left(10^{3}-10^{-4} \mathrm{~Hz}\right)$
}

\author{
R. MICHEL (*), M. MAITROT (**) et G. ASCH (**)
}

Laboratoire d'Electronique, d'Automatique et de Mesures Electriques, Ecole Centrale de Lyon, 36, route de Dardilly, 69730 Ecully, France

(Reçu le 27 mars 1973, révisé le 18 juin 1973)

\begin{abstract}
Résumé. - On a étudié en très basse fréquence $\left(10^{3}-10^{-4} \mathrm{~Hz}\right)$ les propriétés diélectriques du nylon 66 (hexaméthylène adipamide) et mis en évidence des relaxations donnant à $10^{-3} \mathrm{~Hz}$ des capacités de l'ordre de $10^{6} \mathrm{pF} / \mathrm{cm}^{2}$, avec un pourcentage en eau de $5 \%$. Ce phénomène correspondrait à l'accumulation des porteurs au voisinage de la surface. L'application de la théorie de Beaumont et Jacobs ne donne pas de résultats satisfaisants à cause d'un second phénomène de relaxation à plus haute fréquence. Cette relaxation correspond à une polarisation plus faible, associée à une forte diminution de la résistance parallèle lorsque la fréquence augmente. Nous avons interprété les résultats expérimentaux à partir d'un circuit équivalent simple dérivé du modèle de Raleigh. La diffusion semble être la cause principale de la polarisation en haute fréquence. On note que la résistance série des échantillons autour de $100 \mathrm{~Hz}$, et leur capacité série en très basse fréquence $\left(10^{-3} \mathrm{~Hz}\right)$ sont relativement indépendantes du degré d'hydratation.

La concentration des porteurs de charges, que l'on suppose être des ions $\mathrm{H}^{+}$reste constante lorsque l'hydratation varie $\left(c_{0} \simeq 10^{23} / \mathrm{m}^{3}\right)$ mais leur mobilité $\mu$ est une fonction exponentielle de la teneur en eau $\left(\mu_{(5 \%)}=2 \times 10^{-13} \mathrm{~m}^{2} /\right.$ V.s $)$.
\end{abstract}

Abstract. - Dielectric properties of nylon 66 (hexamethylene adipamide) were studied as a function of humidity near $50^{\circ} \mathrm{C}$ at very low frequencies $\left(10^{3}-10^{-4} \mathrm{~Hz}\right)$. We found a relaxation giving near $10^{-3} \mathrm{~Hz}$ a capacitance as high as $10^{6} \mathrm{pF} / \mathrm{cm}^{2}$ for a water content of $5 \%$. Polarization and dielectric absorption are explained in terms of motion of carriers near the surface of the samples. We tried to use the theoretical treatment given by Beaumont and Jacobs, but it failed because of complex relaxation phenomena at higher frequencies. A Raleigh's series model with some improvements fits the experimental results at low frequencies. Higher frequency polarizations $\left(10^{3}-10^{-2} \mathrm{~Hz}\right)$ can be explained by diffusion phenomena.

We found that the concentration $c_{0}$ of charge carriers, presumably $\mathrm{H}^{+}$ions, is constant $\left(c_{0} \simeq 10^{23} / \mathrm{m}^{3}\right)$ but their mobility $\mu$ is an exponential function of water content

$$
\left(\mu_{(5 \%)}=2 \times 10^{-13} \mathrm{~m}^{2} / \mathrm{V} . \mathrm{s}\right) \text {. }
$$

L'étude des propriétés électriques des polyamides hydratés a fait l'objet de travaux assez nombreux, soit à fréquences relativement élevées [1], [2] soit en continu [3] mais rarement aux très basses fréquences [4], [5]. Dans ces conditions on a pensé qu'il pouvait être intéressant de refaire à ces fréquences l'étude d'échantillons déjà examinés à sec [6] afin de suivre l'évolution de leurs propriétés diélectriques en fonction de leur teneur en eau.

1. Dispositif expérimental. - Les mesures ont été faites dans un domaine de fréquences s'étendant de $10^{-5} \mathrm{~Hz}$ à $10^{5} \mathrm{~Hz}$. Une telle étendue de fréquences ne pouvant être explorée par un seul type d'appareil de mesure a donc été divisée, suivant la technique utilisée, en trois parties.

(*) Ecole Centrale de Lyon, 69130 Ecully.

(**) Université Claude-Bernard, 43, boulevard du 11-Novembre-1918, 69621 Villeurbanne.
Entre $100 \mathrm{kHz}$ et $100 \mathrm{~Hz}$, on a utilisé uı pont d'impédance à transformateur Wayne-Kerr B 331. De $100 \mathrm{~Hz}$ à $10^{-1} \mathrm{~Hz}$ on a employé soit un pont d'impédance soit un appareillage basé sur une détection synchrone. Le pont d'impédance du type de celui décrit par Scheiber [7] a été utilisé pour des conductances inférieures à $5 \times 10^{-8}$ mhos et des capacités inférieures à $170 \mathrm{pF}$. L'équilibre du pont est vérifié à l'aide d'un électromètre Keithley $610 \mathrm{C}$, suivi aux fréquences supérieures à $10 \mathrm{~Hz}$ par un détecteur sélectif Général Radio $1232 \mathrm{~A}$. Quand la conductivité dépasse les possibilités de mesure du pont, on a employé un détecteur de phase recevant le signal issu de l'électromètre Keithley. Le découpage synchrone de ce signal est réalisé par des transistors MOS, alternativement saturés et bloqués, commandés par une tension, dite de référence en phase ou en quadrature avec la tension appliquée à l'échantillon. Un filtre passe bas délivre, selon la phase de la tension de référence, une tension proportionnelle soit à la composante $I^{\prime \prime}$ du courant en phase avec la tension appliquée, soit à la compo- 
sante $I^{\prime}$ du courant en quadrature avec cette tension. La constante diélectrique $\varepsilon^{*}=\varepsilon^{\prime}-j \varepsilon^{\prime \prime}$ se calcule alors aisément :

$$
\varepsilon^{\prime}=\frac{A I^{\prime} L}{\omega V \varepsilon_{0} S}, \quad \varepsilon^{\prime \prime}=\frac{A I^{\prime \prime} L}{\omega V \varepsilon_{0} S}
$$

avec : $A$ gain de la détection synchrone,

$\omega, V$ pulsation et amplitude de la tension appliquée,

$L, S$ épaisseur et surface de l'échantillon,

$\varepsilon_{0} \quad$ permittivité absolue du vide.

Dans le troisième domaine de fréquences, de $10^{-1} \mathrm{~Hz}$ à $10^{-5} \mathrm{~Hz}, I^{\prime}$ et $I^{\prime \prime}$ ont été mesurés sur l'enregistrement graphique du courant en fonction de la tension [8]. Afin d'éviter d'éventuelles distorsions d'amplitude, la tension alternative appliquée ne dépasse pas $0,1 \mathrm{~V}$.

La température de la cellule de mesure est régulée à $0,1^{\circ} \mathrm{C}$ près ; la cellule est placée dans une enceinte étanche dont la température légèrement inférieure à celle de la cellule est constante à $0,5^{\circ} \mathrm{C}$ près lorsque la température ambiante varie de $10^{\circ} \mathrm{C}$. Un capteur de chlorure de lithium (Philips) associé à un régulateur permet de mesurer l'humidité et de contrôler, par l'intermédiaire d'un ventilateur, l'admission de vapeur d'eau. La précision de cette régulation est d'environ $0,5 \%$ de degré hygrométrique.

Les échantillons de nylon 66 sont d'origine commerciale (Rhodiacéta) ; ils se présentent sous forme de plaques, obtenues par extrusion, d'épaisseur $0,1 \mathrm{~cm}$. Les électrodes, de surface $3,4 \mathrm{~cm}^{2}$, sont constituées par de l'aluminium déposé sous vide. Le degré de cristallinité est de 0,54 . La quantité d'eau absorbée est déterminée par pesée au dixième de milligramme près.

2. Résultats expérimentaux. - Dans une première série de mesures, l'échantillon a été placé plusieurs semaines dans une atmosphère à $95 \%$ d'humidité relative. L'eau absorbée représente alors environ $5 \%$ du poids de l'échantillon.

L'impédance mesurée peut être représentée soit par l'ensemble en parallèle d'une capacité $C_{\mathrm{p}}$ et d'une résistance $R_{\mathrm{p}}$ soit par le groupement en série d'une capacité $C_{\mathrm{s}}$ et d'une résistance $R_{\mathrm{s}}$. Entre les éléments des montages parallèle et série existent les relations évidentes :

$$
\begin{aligned}
& R_{\mathrm{s}}=\frac{R_{\mathrm{p}}}{1+\left(R_{\mathrm{p}} C_{\mathrm{p}} \omega\right)^{2}} \\
& C_{\mathrm{s}}=\frac{1+\left(R_{\mathrm{p}} C_{\mathrm{p}} \omega\right)^{2}}{R_{\mathrm{p}}^{2} C_{\mathrm{p}} \omega^{2}} .
\end{aligned}
$$

Les figures 1, 2, 3 représentent en fonction de la fréquence les variations de la constante diélectrique $\varepsilon^{\prime}$, de $\operatorname{tg} \delta=\frac{G_{\mathrm{p}}}{C_{\mathrm{p}} \omega}$, de $G_{\mathrm{p}}=\frac{1}{R_{\mathrm{p}}}$ lorsque la température varie, l'humidité de l'atmosphère extérieure demeurant constamment égale à $95 \%$.

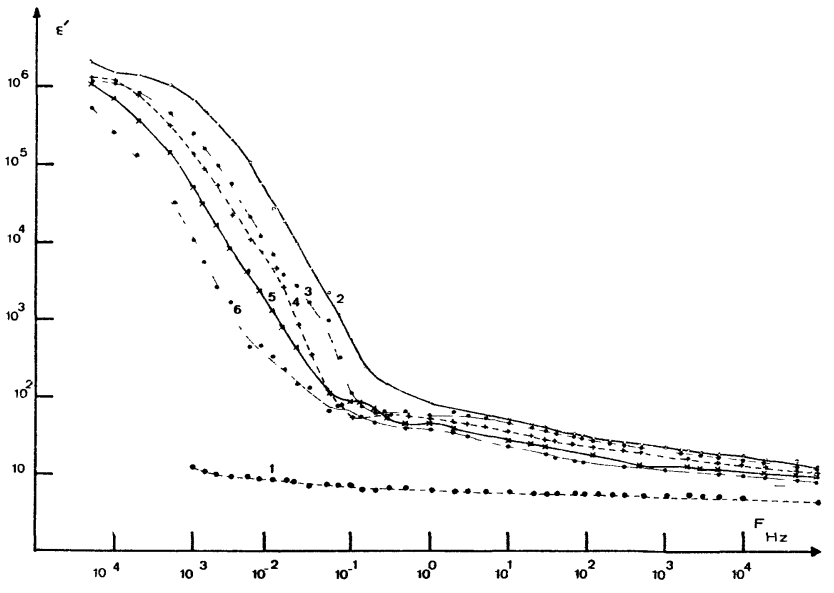

FIG. 1. - Variation de la constante diélectrique du nylon 66 ; échantillon ayant les dimensions suivantes : épaisseur $1 \mathrm{~mm}$, surface $3,37 \mathrm{~cm}^{2}$ :

courbes 1 , nylon sec, 2 et 3 nylon humide à $50^{\circ} \mathrm{C}$, courbe $4,55^{\circ} \mathrm{C}$, degré d'humidité extérieure $95 \%$, courbe $5,65^{\circ} \mathrm{C}$, degré d'humidité extérieure $95 \%$, courbe $6,76^{\circ} \mathrm{C}$, degré d'humidité extérieure $95 \%$.

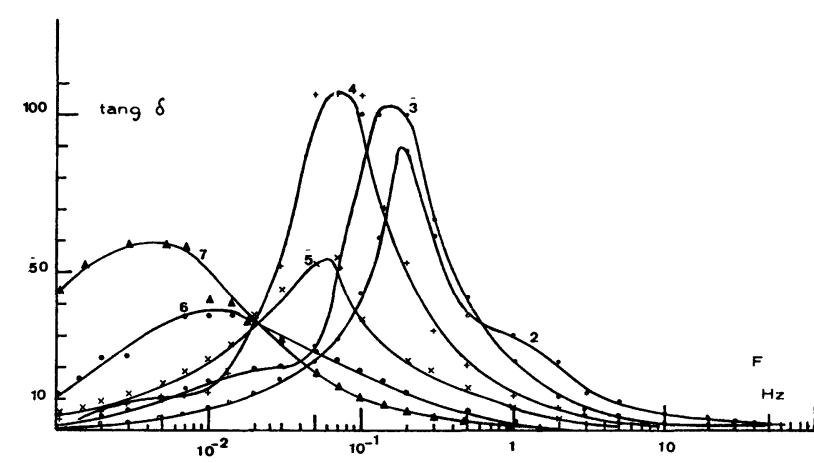

FIG. 2. - Variation de tg $\delta$ avec la fréquence (mêmes conditions que Fig. 1) sauf courbe 7, humidité relative $80 \%$ et température $50^{\circ} \mathrm{C}$.

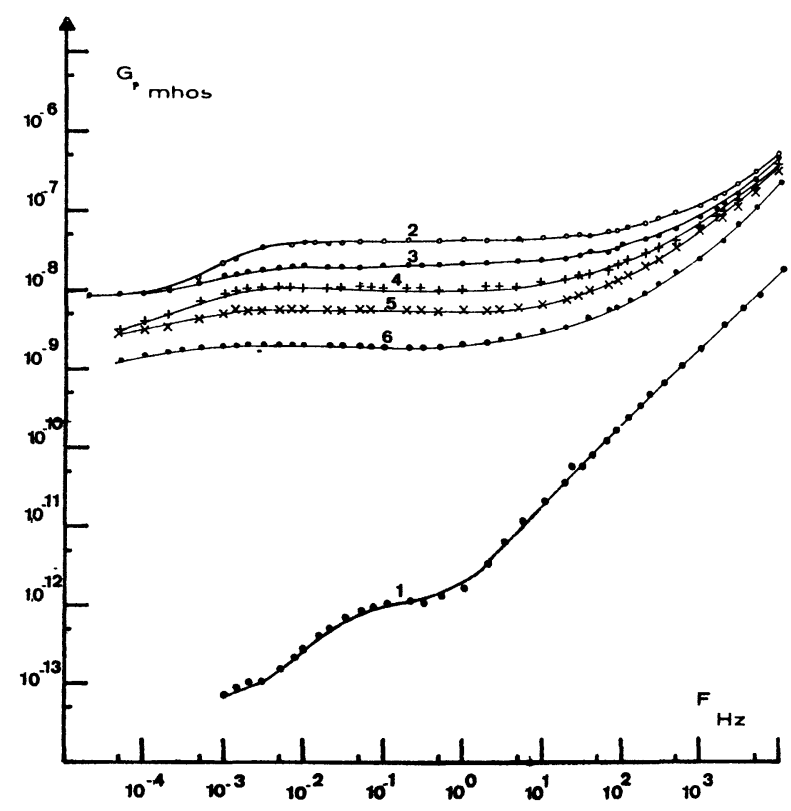

Fig. 3. - Variation de $G_{\mathrm{p}}$ avec la fréquence. 
A $50^{\circ} \mathrm{C}$, on observe une augmentation progressive et régulière de $\varepsilon^{\prime}$, proportionnel à $C_{\mathrm{p}}$, et de $G_{\mathrm{p}}$, à mesure que l'échantillon d'abord sec, se sature en humidité (courbes 1 , puis 3 et 2 des Fig. 1 et 3 ). En même temps on observe l'apparition d'un pic de $\operatorname{tg} \delta$ vers $0,3 \mathrm{~Hz}$ de valeur voisine de 100 (courbes 3 et 2 , Fig. 2). Puis à mesure que la température augmente on observe à fréquence donnée, et à hygrométrie ambiante constante une diminution de $\varepsilon^{\prime}$ et de $G_{\mathrm{p}}$. Le maximum de $\operatorname{tg} \delta$ se déplace vers les basses fréquences (Fig. 2 courbes 4, 5, 6). Il n'existe pas sur l'échantillon sec.

A température constante on observe en fonction de la fréquence les évolutions suivantes :

- La constante diélectrique $\varepsilon^{\prime}$, augmente très lentement lorsque la fréquence passe de $10^{5}$ à $1 \mathrm{~Hz}$ puis croît rapidement avec une pente voisine de 2 en coordonnées logarithmiques pour atteindre vers $10^{-3} \mathrm{~Hz}$ un palier de l'ordre de $10^{6}$ correspondant à une capacité $C_{\mathrm{p}}$ de $0,4 \mu \mathrm{F}$. Ce palier est décalé vers les basses fréquences lorsque la température s'élève (Fig. 1, courbes 2, 4, 5, 6).

- La conductance $G_{\mathrm{p}}$ décroît jusque vers $10 \mathrm{~Hz}$ puis présente un palier jusqu'à $10^{-3} \mathrm{~Hz}$, pour décroître à nouveau au-delà (Fig. 3, courbes $2,4,5,6$ ).

- La tangente de l'angle de perte, $\operatorname{tg} \delta$, présente un maximum dont la valeur décroît de $100\left(50^{\circ} \mathrm{C}\right)$ à $30\left(76^{\circ} \mathrm{C}\right)$ lorsque la température augmente.

La représentation sous forme série de l'impédance

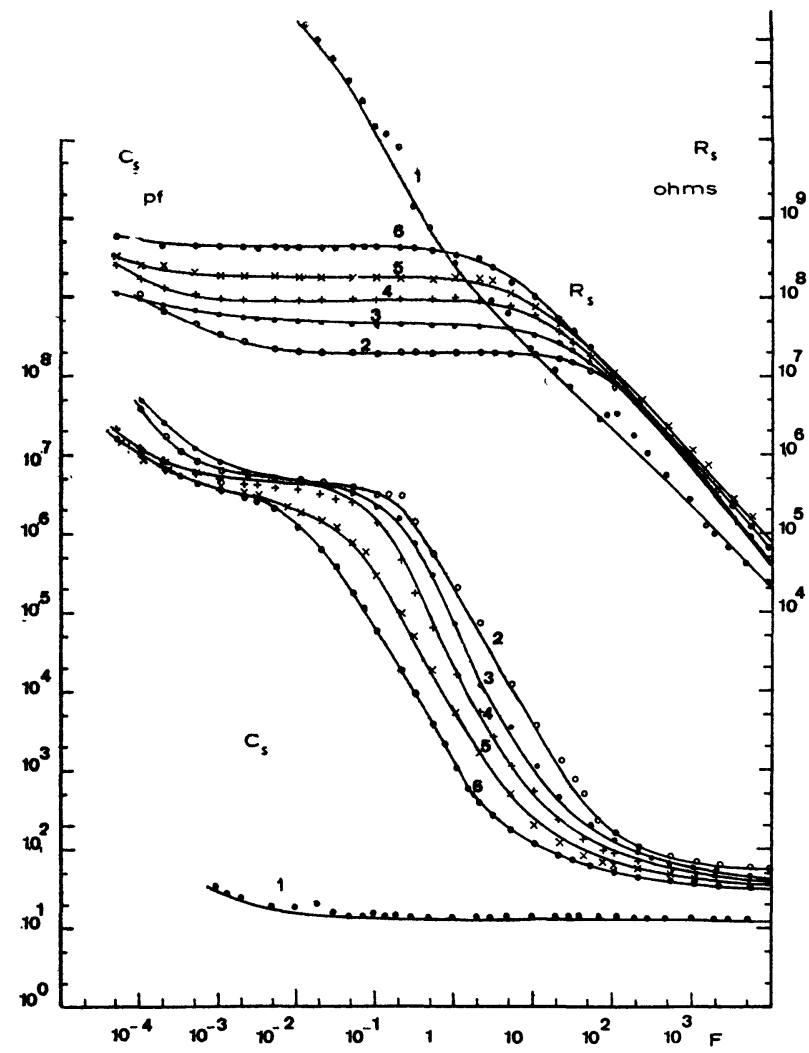

FIg. 4. - Variation de la capacité et de la résistance série (mêmes conditions que Fig. 1). de l'échantillon est indiquée figure 4. On y constate que :

- L'augmentation de $C_{\mathrm{s}}$ a lieu à des fréquences nettement plus élevées que pour $C_{\mathrm{p}}$ et que le palier est bien plus étendu.

- La résistance $R_{\mathrm{s}}$, quand la fréquence diminue jusqu'environ $10 \mathrm{~Hz}$, augmente indépendamment de la température et de l'hygrométrie puis présente un palier de valeur d'autant plus grande que la tempéra) ture est plus haute. Les figures 5 et 6 représentent la variation des éléments constitutifs de la représentation parallèle $\left(C_{\mathrm{p}}, G_{\mathrm{p}}\right)$ et de la représentation série $\left(C_{\mathrm{s}}, R_{\mathrm{s}}-\right.$ de l'échantillon maintenu à température constante $\left(50^{\circ} \mathrm{C}\right)$ avec une humidité variant de 0 à $5 \%$. La capacité $C_{\mathrm{p}}$ ne présente pas de palier aux fréquences étudiées, contrairement à $C_{\mathrm{s}}$; la conductance $G_{\mathrm{p}}$ augmente avec l'humidité et décroît quand la fréquence diminue ; par contre $R_{\mathrm{s}}$ présente aux fréquences basses un palier d'autant plus étendu que l'humidité est plus forte ; aux fréquences élevées $R_{\mathrm{s}}$ est indépendant de l'humidité et varie avec une pente voisine de 1 .

En fonction de l'humidité, la résistance série évolue différemment selon la fréquence (Fig. 7). En très basse fréquence $\left(10^{-3} \mathrm{~Hz}\right)$ le logarithme de $R_{\mathrm{s}}$ est

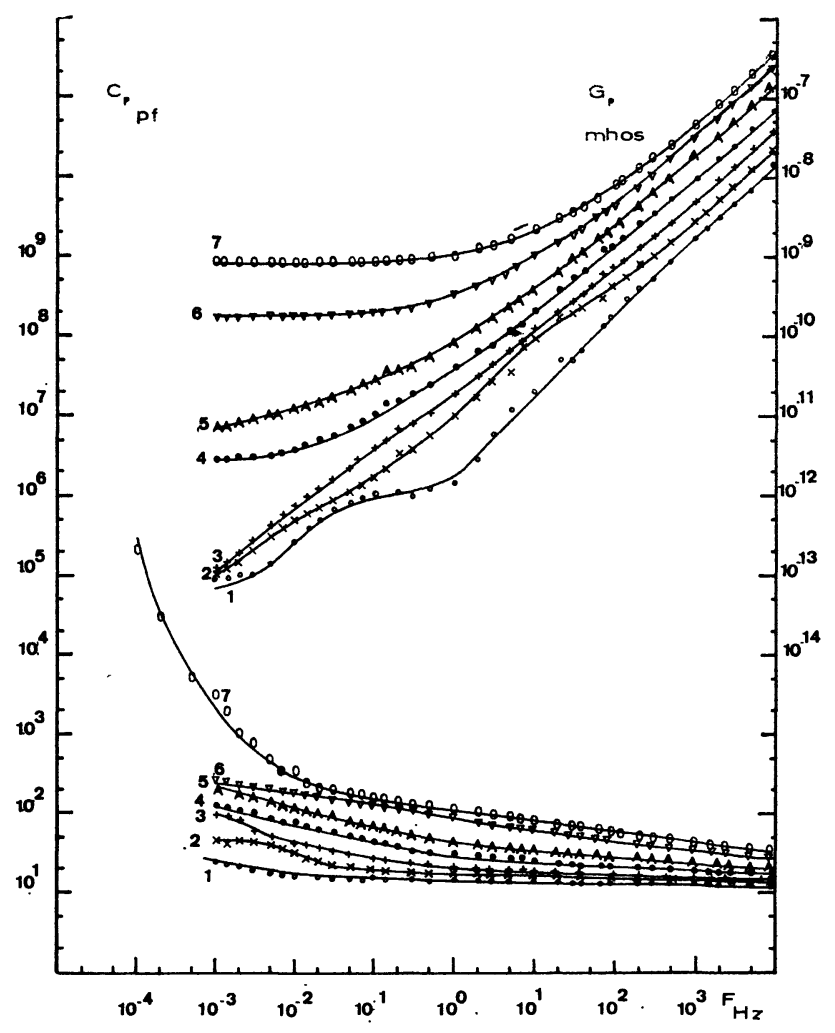

Fig. 5. - Variation de la capacité et de la conductance parallèle d'un échantillon à humidité variable à $T=50^{\circ} \mathrm{C}$ :

1) échantillon sec,

2) humidité $2,7 \%$ en poids de l'échantillon,

3) humidité $5,5 \%$ en poids de l'échantillon,

4) humidité $1,34 \%$ en poids de l'échantillon,

5) humidité $2,03 \%$ en poids de l'échantillon,

6) humidité $2,7 \%$ en poids de l'échantillon,

7) humidité $5 \%$ en poids de l'échantillon. 


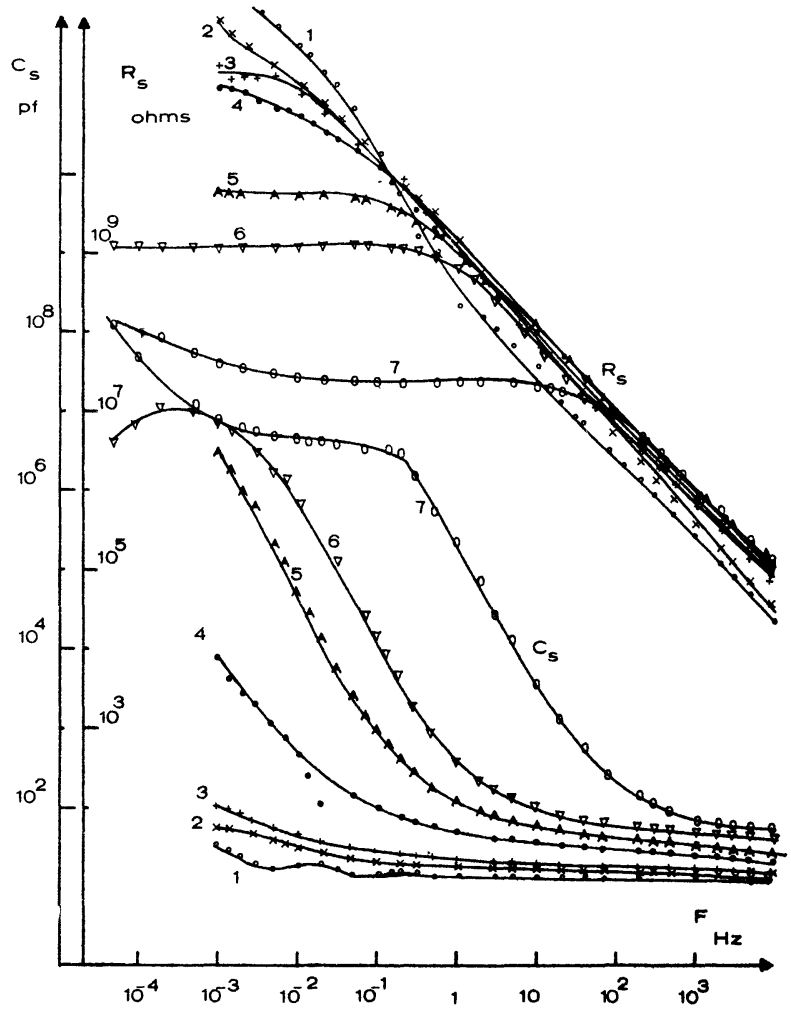

Fig. 6. - Variation de la capacité et de la résistance série du nylon 66 , à $50^{\circ} \mathrm{C}$ :
1) échantillon sec,
2) humidité $2,7 \%$ en poids de l'échantillon,
3) humidité $5,5 \%$ en poids de l'échantillon,
4) humidité $1,34 \%$ en poids de l'échantillon,
5) humidité $2,03 \%$ en poids de l'échantillon,
6) humidité $2,7 \%$ en poids de l'échantillon,
7) humidité $5 \%$ en poids de l'échantillon

fonction linéaire du pourcentage d'humidité $m$. A plus haute fréquence $(1 \mathrm{~Hz}) R_{\mathrm{s}}$ croît, présente un palier, puis décroît exponentiellement. On observe un effet de saturation pour une humidité de $5 \%$.

3. Recherche d'un modèle théorique. - 3.1 MoDÈLE DE BeAumont ET JACOBS [9]. - Les résultats expérimentaux, en particulier les valeurs très élevées de la capacité aux très basses fréquences, indiquent que les phénomènes observés sont liés à l'accumulation de charges au voisinage des électrodes. On a donc tout d'abord comparé les résultats de mesure à ceux que prévoit la théorie de Beaumont et Jacobs pour un milieu homogène, avec un seul type de porteurs, sans recombinaison et avec blocage partiel; on a dans ce cas les expressions suivantes de $C_{\mathrm{p}}$ et $G_{\mathrm{p}}$ :

$$
\begin{aligned}
C_{\mathrm{p}}= & C_{\mathrm{p}}^{\prime}+C_{\infty}=\left(\frac{\pi k T}{\varepsilon c_{0}}\right)^{1 / 2} \frac{4 \sigma^{2}}{\omega^{2} L^{2} e} \\
& \times\left[1+\frac{\pi k T}{\varepsilon c_{0}} \frac{4 \sigma^{2}}{\omega^{2} L^{2} e^{2}}(2+\rho)^{2}\right]^{-1}+C_{\infty}
\end{aligned}
$$

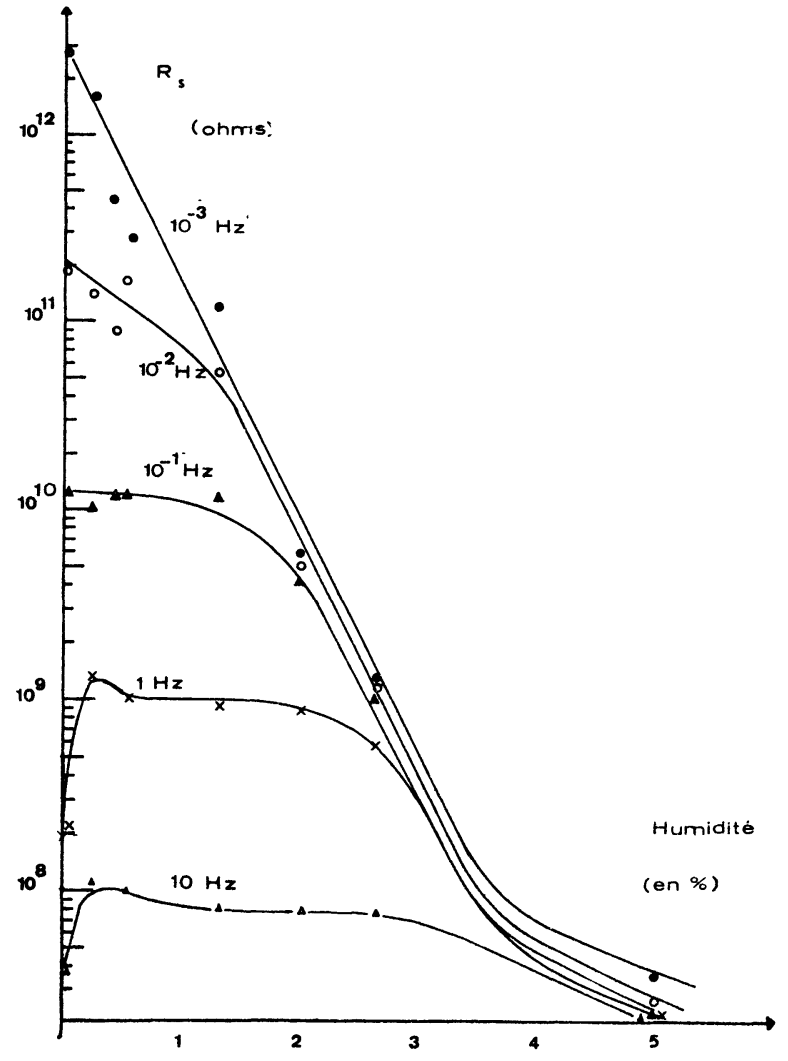

Fig. 7. - Variation de $R_{\mathrm{s}}=R_{0}$ avec la contenance en humidité de l'échantillon.

$$
\begin{aligned}
G_{\mathrm{p}}=G_{0}-\frac{8 \sigma^{3} \pi k T(2+\rho)}{L^{3} e^{2} c_{0} \varepsilon \omega^{2}} & \\
& \times\left[1+\frac{\pi k T}{\varepsilon c_{0}} \frac{4 \sigma^{2}(2+\rho)^{2}}{\omega^{2} L^{2} e^{2}}\right]^{-1}
\end{aligned}
$$

où : $e$ charge de l'électron,

$\varepsilon \quad$ permittivité du milieu sans charge d'espace,

$\rho$ coefficient de blocage,

$\sigma$ conductivité de l'échantillon sans charge d'espace,

$L$ épaisseur,

$c_{0}$ concentration en porteurs,

$k$ constante de Boltzmann,

$T$ température absolue,

$C_{\infty}$ capacité de l'échantillon à très haute fréquence,

$C_{\mathrm{p}}^{\prime}$ capacité de charge d'espace.

Il n'est pas possible de faire coïncider ces expressions avec les résultats expérimentaux : en effet, les valeurs de $\rho$ et $c_{0}$ que l'on peut calculer à partir d'une courbe de variation de $C_{\mathrm{p}}$ sont très différentes de celles que l'on obtient à partir de la courbe représentative de la conductance $G_{\mathrm{p}}$ du même échantillon. Il semble donc que ce modèle ne convienne pas lorsque, comme cela doit être le cas ici, d'autres effets de relaxation se superposent à l'effet de charge d'espace. 
3.2 Modèle De Raleigh [11]. - 3.2.1 Cas d'un blocage total. - Raleigh a remarqué que, d'après Mac Donald [12] et Friauf [10], lors d'un blocage total des électrodes la capacité $C_{\mathrm{p}}$ et la résistance $R_{\mathrm{p}}$ du circuit parallèle équivalent au diélectrique peuvent s'écrire :

$$
\begin{aligned}
& C_{\mathrm{p}}=C_{0} \frac{1}{1+\left(\omega R_{0} C_{0}\right)^{2}} \\
& R_{\mathrm{p}}=R_{0}\left[1+\frac{1}{\left(\omega R_{0} C_{0}\right)^{2}}\right]
\end{aligned}
$$

où $R_{0}$ est la résistance de l'échantillon en l'absence de blocage,

$C_{0}$ est la capacité limite en basse fréquence $\left(C_{0}=C_{\infty} \cdot L / L_{\mathrm{D}}\right)$,

$L_{\mathrm{D}}$ étant la longueur de Debye et $C_{\infty}$ la capacité aux fréquences élevées.

Le circuit série, équivalent à l'ensemble $R_{\mathrm{p}} C_{\mathrm{p}}$ en parallèle, a pour éléments $R_{\mathrm{s}}$ et $C_{\mathrm{s}}$ donnés par les formules (1) et (2) qui, pour les expressions (5) et (6) de $C_{\mathrm{p}}$ et $R_{\mathrm{p}}$ se réduisent à

$$
C_{\mathrm{s}}=C_{0} \quad \text { et } \quad R_{\mathrm{s}}=R_{0} .
$$

Dans le cas d'un blocage total, le circuit série équivalent au diélectrique est donc constitué d'une résistance $R_{0}$ et d'une capacité $C_{0}$ indépendantes de la fréquence (Fig. $8 a, 8 b$ ).

a)

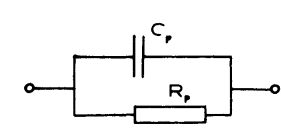

b)
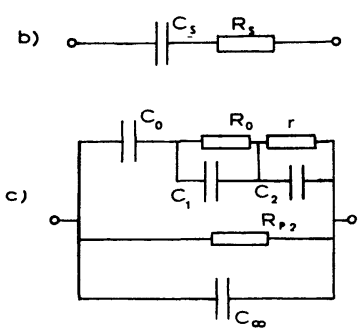

FIG. 8. - Schéma équivalent au cristal. Représentation asymptotique de $R_{\mathrm{s}}$ et $C_{\mathrm{s}}$; fréquences de coupure.

On se propose d'examiner si un tel circuit équivalent demeure valable dans le cas d'un blocage partiel.

3.2.2 Cas d'un blocage partiel. - On a établi précédemment (6), à partir des résultats de Beaumont et Jacobs et de Vanderschueren [13] les expressions des éléments $C_{\mathrm{p}}$ et $R_{\mathrm{p}}$ du circuit parallèle équivalent à l'échantillon :

$$
\begin{aligned}
& C_{\mathrm{p}}=C_{\infty}\left(1+\frac{l(A)}{(1+\rho / 2)^{2}+\omega^{2}\left(l(A) \omega_{\mathrm{c}}^{-1}\right)^{2}}\right) \\
& \frac{1}{R_{\mathrm{p}}}=\frac{1}{R_{0}}\left(1-\frac{(1+\rho / 2)}{(1+\rho / 2)^{2}+\omega^{2}\left(l(A) \omega_{\mathrm{c}}^{-1}\right)^{2}}\right) .
\end{aligned}
$$

Dans ces expressions: $l(A)$ est la fonction de Langevin du paramètre $A=L / L_{\mathbf{D}}$; quand $L / L_{\mathbf{D}} \gg 1$, $l\left(L / L_{\mathrm{D}}\right)$ est très voisin de $L / L_{\mathrm{D}}$.

$\omega_{\mathrm{c}}$ est la pulsation caractéristique de l'échantillon :

$$
\omega_{\mathrm{c}}=\frac{1}{R_{0} C_{\infty}} .
$$

En posant : $C_{0}=C_{\infty} \frac{l(A)}{(1+\rho / 2)^{2}}$, les expressions (7) et (8) se récrivent, en négligeant $C_{\infty}$ devant $C_{0}$ :

$C_{\mathrm{p}}=\frac{C_{0}}{1+\omega^{2} R_{0}^{2} C_{0}^{2}(1+\rho / 2)^{2}}$

$\frac{1}{R_{\mathrm{p}}}=\frac{1}{R_{0}} \frac{\omega^{2} R_{0}^{2} C_{0}^{2}(1+\rho / 2)^{2}}{1+\omega^{2} R_{0}^{2} C_{0}^{2}(1+\rho / 2)^{2}}+$

$$
+\frac{1}{R_{0}} \frac{\rho / 2}{(1+\rho / 2)\left[1+\omega^{2} R_{0}^{2} C_{0}^{2}(1+\rho / 2)^{2}\right]} .
$$

Si le coefficient $\rho$ est faible, il existe un intervalle de fréquences correspondant à

$$
\omega \gg \frac{1}{R_{0} C_{0}}\left[\frac{\rho / 2}{(1+\rho / 2)^{3}}\right]^{1 / 2}=\omega_{\mathrm{c}}^{\prime}
$$

pour lesquelles le premier terme de (10) est prépondérant ; pour ce domaine de fréquences :

$$
\begin{aligned}
& C_{\mathrm{p}}=C_{0} \frac{1}{1+\omega^{2} R_{0}^{2} C_{0}^{2}(1+\rho / 2)^{2}} \\
& R_{\mathrm{p}}=R_{0} \frac{1+\omega^{2} R_{0}^{2} C_{0}^{2}(1+\rho / 2)^{2}}{\omega^{2} R_{0}^{2} C_{0}^{2}(1+\rho / 2)^{2}} \simeq R_{0} .
\end{aligned}
$$

Dans cette gamme de fréquences $\left(\omega \gg \omega_{\mathrm{c}}^{\prime}\right)$ apparaît une autre pulsation de coupure

$$
\omega_{\mathrm{c}}^{\prime \prime}=\frac{1}{R_{0} C_{0}}\left(1+\frac{\rho}{2}\right)
$$

déterminant les variations de $C_{\mathrm{p}}$ et $\boldsymbol{R}_{\mathrm{p}}$.

On remarque que

$$
\omega_{\mathrm{c}}^{\prime}=\omega_{\mathrm{c}}^{\prime \prime}\left[\frac{\rho / 2}{(1+\rho / 2)}\right]^{1 / 2}
$$

soit

$$
\omega_{\mathrm{c}}^{\prime}<\omega_{\mathrm{c}}^{\prime \prime} .
$$

Pour $\omega \gg \omega_{\mathrm{c}}^{\prime \prime}$

$$
C_{\mathrm{p}}=C_{0}\left(\frac{\omega_{\mathrm{c}}^{\prime \prime}}{\omega}\right)^{2}, \quad R_{\mathrm{p}}=R_{0}
$$

et d'après les formules (1) et (2)

$$
C_{\mathrm{s}}=\left(1+\frac{\rho}{2}\right)^{2} C_{0}, \quad R_{\mathrm{s}}=R_{0} .
$$


Dans le cas où $\omega_{\mathrm{c}}^{\prime} \ll \omega \ll \omega_{\mathrm{c}}^{\prime \prime}$

$$
\begin{array}{ll}
C_{\mathrm{p}}=C_{0}, & R_{\mathrm{p}}=R_{0}\left(\frac{\omega_{\mathrm{c}}^{\prime \prime}}{\omega}\right)^{2} \\
C_{\mathrm{s}}=C_{0}, & R_{\mathrm{s}}=R_{0}\left(1+\frac{\rho}{2}\right)^{2} .
\end{array}
$$

Lorsque $\omega \ll \omega_{c}^{\prime}$ on peut mettre en évidence une seconde pulsation caractéristique :

$$
\omega_{\mathrm{c}}^{\prime \prime \prime}=\omega_{\mathrm{c}}^{\prime \prime} \times \frac{\rho}{2}=\omega_{\mathrm{c}}^{\prime} \times\left(\left(\frac{\rho}{2}\right)\left(1+\frac{\rho}{2}\right)\right)^{1 / 2} .
$$

Lorsque $\omega \ll \omega_{\mathrm{c}}^{\prime \prime \prime} \ll \omega_{\mathrm{c}}^{\prime}$ on a :

$$
\begin{gathered}
C_{\mathrm{p}}=C_{0} ; \quad R_{\mathrm{p}}=R_{0} \frac{(1+\rho / 2)}{\rho / 2} \\
C_{\mathrm{s}}=C_{0}\left[\frac{(\rho / 2)^{2}}{\omega^{2} R_{0}^{2} C_{0}^{2}(1+\rho / 2)^{2}}\right] ; \quad R_{\mathrm{s}}=R_{0} \frac{1+\rho / 2}{\rho / 2} .
\end{gathered}
$$

Expérimentalement on détermine $\omega_{\mathrm{c}}^{\prime}$ sur la courbe de $G_{\mathrm{p}}=1 / R_{\mathrm{p}}$ (palier très basse fréquence) et $\omega_{\mathrm{c}}^{\prime \prime}$ sur la courbe de $C_{\mathrm{p}}$ ou de $\varepsilon^{\prime}$. Pour la courbe 2 des figures 1 et 3 , les fréquences mesurées sont $10^{-4}$ et $1,6 \times 10^{-3} \mathrm{~Hz}$, d'où $\omega_{\mathrm{c}}^{\prime}=6 \times 10^{-4} \mathrm{rad} / \mathrm{s}$ et $\omega_{\mathrm{c}}^{\prime \prime}=10^{-2} \mathrm{rad} / \mathrm{s}$. $\omega_{\mathrm{c}}^{\prime \prime \prime}$ n'est pas accessible. Un comportement analogue a été observé plus nettement sur la polyglycine humide.

4. Discussion du comportement en très basse fréquence. - Les résultats expérimentaux en accord qualitatif avec l'analyse précédente permettent tout d'abord de mesurer $R_{0}$ et $C_{0}$, $\omega_{\mathrm{c}}^{\prime}$ et $\omega_{\mathrm{c}}^{\prime \prime}$ et d'atteindre la valeur de $\rho$ par différentes méthodes. Pour la courbe 2, figures 1 et 3 les pulsations de coupure mesurées sont $\omega_{\mathrm{c}}^{\prime \prime}=10^{-2} \mathrm{rad} / \mathrm{s}$ et $\omega_{\mathrm{c}}^{\prime}=6 \times 10^{-4} \mathrm{rad} / \mathrm{s}$; on en déduit la valeur de $\rho$ d'après la relation (13) : on trouve $\rho \simeq 10^{-2}$.

D'autre part, pour ce même échantillon on mesure $R_{0} \simeq 2 \times 10^{7} \Omega, C_{0} \simeq 5 \times 10^{-6} \mathrm{~F},\left(C_{0} R_{0}\right)^{-1} \simeq 10^{-2}:$ la comparaison de cette valeur avec $\omega_{\mathrm{c}}^{\prime \prime}$ montre un bon accord, et conduirait également à une faible valeur de $\rho$.

Par contre, si l'on compare la résistance série aux très basses fréquences $R_{\mathrm{s}}=R_{0}\left(\frac{1+\rho / 2}{\rho / 2}\right)$ avec la résistance à une pulsation supérieure à $\omega_{\mathrm{c}}^{\prime \prime}, R_{\mathrm{s}}=R_{0}$, on en déduit une valeur de $\rho$ de l'ordre de 0,4 . Alors que les fréquences de coupure correspondent bien à ce que la théorie précédente permet de prévoir, il y a cependant une certaine indétermination sur les valeurs de $\rho$. Ceci pourrait s'expliquer si $\rho$ était une fonction de la fréquence.

- Lorsque $\omega>\omega_{\mathrm{c}}^{\prime}$ les expressions (7) et (8) indiquent une relaxation du type de Debye. On peut écrire :

$$
C_{\mathrm{p}}-C_{\infty}=\frac{C_{0}-C_{\infty}}{1+\omega^{2} \tau^{2}}
$$

$$
\begin{aligned}
G_{\mathrm{p}} & =G_{0} \frac{\omega^{2} \tau^{2}}{1+\omega^{2} \tau^{2}}\left(1+\frac{\rho}{2}\right) \\
C_{0} & =\frac{L}{L_{\mathrm{D}}} \frac{C_{\infty}}{(1+\rho / 2)^{2}} \\
\tau & =\frac{L}{L_{\mathrm{D}}} \frac{1}{\omega_{\mathrm{c}}(1+\rho / 2)} \\
\omega_{\mathrm{c}} & =\frac{\sigma_{\infty}}{\varepsilon_{\infty}}=\frac{1}{R_{0} C_{\infty}} \\
\omega_{\mathrm{M}} & =\omega_{\mathrm{c}}\left(\frac{L}{L_{\mathrm{D}}}\right)^{1 / 2} .
\end{aligned}
$$

A $\omega_{\mathrm{M}}$ correspond le maximum de $\operatorname{tg} \delta$.

Nous considérons que $\rho \simeq 10^{-2}$; dans le domaine de fréquences considéré $\left(\omega>\omega_{\mathrm{c}}^{\prime \prime}\right)$ on a

$$
\operatorname{tg} \delta_{\max }=\frac{1}{2}\left(\frac{L}{L_{\mathrm{D}}}\right)^{1 / 2}
$$

l'application de ces formules aux résultats expérimentaux permet le calcul de $L / L_{\mathrm{D}}$. Par exemple pour la courbe 2 figure 3 ,

$$
\operatorname{tg} \delta_{\max }=80 \text { et } \frac{L}{L_{\mathrm{D}}} \simeq 2,5 \times 10^{4} .
$$

Portant cette valeur dans l'expression de $C_{0}$ on constate que la valeur calculée pour $C_{\infty}$ est beaucoup plus grande que celle de la capacité géométrique; nous remplacerons alors $C_{\infty}$ par cette valeur $C_{1}$.

D'autre part, la mesure de $\omega_{\mathrm{M}}$ nous fournit également la valeur de $\omega_{\mathrm{c}}=\frac{1}{R_{0} C_{\infty}}:$ pour obtenir un bon accord il faut là aussi remplacer $C_{\infty}$ par $C_{1}$.

Enfin, on peut calculer d'autre part la valeur de $L_{\mathbf{D}}$ en écrivant :

$$
C_{0}=\frac{1}{L_{\mathrm{D}}} \frac{\varepsilon_{\infty} S}{(1+\rho / 2)^{1 / 2}} \text { puisque } C_{\infty}=\varepsilon_{\infty} \frac{S}{L} .
$$

Donnant à $\varepsilon_{\infty}$ la valeur $3,33 \times 8,86 \times 10^{-12}$ [18] on évalue $L_{\mathrm{D}}$, et de $L_{\mathrm{D}}$ on tire la valeur de $L$ à partir de $\operatorname{tg} \delta$.

On trouve $L_{\mathrm{D}} \simeq 4 \times 10^{-5} \mathrm{~m}$.

$$
\frac{L}{L_{\mathrm{D}}} \simeq(160)^{2} \text { d'ou } L \simeq 10^{-4} \mathrm{~m} .
$$

Ceci correspond bien à l'épaisseur de la couche dont la capacité est représentée par $C_{1}$.

De la valeur de $L_{\mathrm{D}}$ et de son expression

$$
L_{\mathrm{D}}=\left(\frac{\varepsilon k T}{2 c_{0} e^{2}}\right)^{1 / 2}
$$

on peut déduire une estimation de la concentration en porteurs : $c_{0} \simeq 5 \times 10^{23} / \mathrm{m}^{3}$.

En fait, l'échantillon n'est pas homogène et cette 
concentration correspond à celle de la région la plus résistante.

Le palier de capacité varie peu avec la teneur en eau : par conséquent la densité des porteurs ne varie pas non plus beaucoup ; c'est leur mobilité qui varie exponentiellement avec le pourcentage d'humidité $m$. Elle atteint une valeur de l'ordre de $0,26 \times 10^{-12} \mathrm{~m}^{2} / \mathrm{V} . \mathrm{s}$ pour $5 \%$ d'eau.

$$
\mu=\mu_{0} \mathrm{e}^{m \alpha} \text { et par conséquent }
$$

$$
\sigma=\sigma_{0} \mathrm{e}^{m \alpha}
$$

$\alpha$ est une constante voisine de $1(\alpha \simeq 1,04)$ et correspond à l'échantillon sec. Cette dernière formule est semblable à celle de Seanor : cependant Seanor la déduit d'une variation du nombre des porteurs dont l'énergie de création diminuerait par suite de l'augmentation de la constante diélectrique avec la proportion d'eau. Nous pensons qu'en fait seule la mobilité dépend de cette proportion.

Pour interpréter le nombre constant des porteurs, on peut calculer la concentration en groupements amine du polymère et la comparer à la densité des porteurs : il y a environ 100 fois moins de porteurs que de groupements amine. Le degré de polymérisation du nylon étant voisin de 100 , il y aurait seulement un porteur par bout de chaîne quand ce nombre est saturé : le mécanisme de la conduction apparaît alors, l'eau se fixant par liaison hydrogène sur le proton d'un groupement amine ou sur l'oxygène d'un groupement carboxyle, en particulier en bout de chaîne : ainsi le nombre de charges assurant la conduction dans le polymère serait relié à sa structure, l'eau jouant seulement un rôle de plastifiant par suite de l'augmentation de la constante diélectrique et permettant un passage plus aisé des porteurs d'une chaîne à l'autre.

Aux plus fortes humidités, la formule (20) est moins bien vérifiée, et conformément aux résultats de King et Medley [14] on observe un effet de saturation. La nature des porteurs est très probablement protonique car on comprendrait mal cet effet de saturation si l'eau agissait seulement comme un donneur d'impuretés électroniques.

On peut enfin comparer ces résultats à ceux de Goffaux [5] concernant du nylon à plus faible température $\left(25^{\circ} \mathrm{C}\right)$ : il obtient une pente plus faible de la capacité parallèle en fonction de la fréquence, mais le rapport de $10^{6}$ entre la permittivité à $10^{-3} \mathrm{~Hz}$ et la permittivité infinie est à peu près le même que pour nos échantillons. Cependant ces résultats concernent peut-être une région de températures inférieures à celles du point de transition vitreuse du nylon.

5. Relaxation « haute fréquence ». - Nous devons donc introduire la capacité $C_{1}$ précédente en parallèle sur $R_{0}$ dans le circuit équivalent à l'échantillon. Cette capacité $C_{1}$ pourrait correspondre à un effet MaxwellWagner entre les régions résistantes et peu résistantes mais n'est cependant pas directement liée au rapport de cristallinité (environ 0,5 ). Elle peut être due à la présence d'une couche superficielle perturbée lors de la fabrication par extrusion de la plaque, elle peut aussi être liée à des phénomènes de diffusion à des interfaces «internes ». On peut proposer pour représenter ce comportement le circuit équivalent de la figure $8 c$. Dans ce circuit, la capacité $C_{0}$ est la capacité de charge d'espace, $R_{0}$ la résistance mesurée précédemment, correspondant à une couche de capacité $C_{1}$; $C_{2}$ et $r$ sont la capacité et la résistance des régions peu résistantes, probablement amorphes ; $\boldsymbol{R}_{\mathrm{p}_{2}}$ est la résistance aux fréquences inférieures à $\omega_{\mathrm{c}}^{\prime}$ et $C_{\infty}$ la capacité géométrique.

On obtient alors les formules suivantes pour la résistance série et la capacité série $C_{\mathrm{s}}$ équivalentes à l'échantillon : nous ferons l'approximation :

$$
C_{2}^{2} r^{2} \omega^{2} \ll 1, \quad r \ll R_{0} \quad \text { et } \quad C_{2} \ll C_{1}
$$

$$
\begin{aligned}
& R_{\mathrm{s}}=\frac{R_{0}}{1+\omega^{2} R_{0}^{2} C_{1}^{2}}+\frac{r}{1+\omega^{2} r^{2} C_{2}^{2}} \\
& C_{\mathrm{s}}=C_{0} \frac{1+C_{1}^{2} R_{0}^{2} \omega^{2}}{1+C_{1}^{2} R_{0}^{2} \omega^{2}+C_{0} \omega\left(C_{1} R_{0}^{2} \omega+C_{2} r^{2} \omega\left(1+C_{1}^{2} R_{0}^{2} \omega^{2}\right)\right)} .
\end{aligned}
$$

Enfin nous ne pouvons déterminer $r$ à partir des résultats expérimentaux; il faudrait atteindre pour l'obtenir, un palier HF à des fréquences bien supérieures à $100 \mathrm{kHz}$ : cette résistance $r$ est certainement faible par rapport à $\boldsymbol{R}_{\mathbf{0}}$.

On pourra donc toujours écrire

$$
R_{\mathrm{s}}=\frac{R_{0}}{1+\omega^{2} R_{0}^{2} C_{1}^{2}}+r
$$

Cherchons les fréquences de coupure de $R_{\mathrm{s}}$ et $C_{\mathrm{s}}$ :

$$
R_{\mathrm{s}}=\frac{R_{0}}{2} \text { pour } \omega_{2}^{\prime}=\frac{1}{R_{0} C_{1}}
$$

$R_{\mathrm{s}}=2 r$ pour $\omega_{1}^{\prime}=\frac{1}{R_{0} C_{1} \sqrt{r / R_{0}}}=\omega_{2}^{\prime}\left(\frac{R_{0}}{r}\right)^{1 / 2}$ 
et de même :

$$
\begin{aligned}
& C_{\mathrm{s}}=\frac{C_{0}}{2} \text { pour } \quad \omega_{2}=\frac{1}{R_{0} C_{1}\left(C_{0} / C_{1}\right)^{1 / 2}} \\
& C_{\mathrm{s}}=C_{1} \quad \text { pour } \quad \omega_{1}=\omega_{2}^{\prime}=\frac{1}{R_{0} C_{1}} .
\end{aligned}
$$

Le tableau I permet de comparer les résultats expérimentaux à ce modèle simple. On constate que $\omega_{1} / \omega_{2}^{\prime}$ est bien voisin de 1 , que le rapport $\left(\omega_{1} / \omega_{2}\right)^{2}$ est sensiblement égal à $C_{0} / C_{1}$. Mais $\omega_{1}$ est voisin de $1 / 2 R_{0} C_{1}$.

Le tableau II montre bien la concordance entre $R_{\mathrm{s}}$ en « haute fréquence » et la quantité $1 / 2 C_{1} \omega$.

\begin{tabular}{|c|c|c|c|c|c|c|c|c|c|c|c|c|c|}
\hline Courbes $n^{\circ}$ & $\begin{array}{c}C_{0} \\
\text { serie }\end{array}$ & $\begin{array}{l}C_{1} \\
\mathrm{pF}\end{array}$ & & $R_{0} \Omega$ & $\frac{1}{R_{0} C_{1}}$ & $\begin{array}{c}\omega_{1} \\
\mathrm{rad} / \mathrm{s}\end{array}$ & $\begin{array}{c}\omega_{2} \\
\mathrm{rad} / \mathrm{s}\end{array}$ & & $\left.\frac{\omega_{1}}{\omega_{2}}\right)^{2}$ & & $\frac{C_{0}}{C_{1}}$ & $\begin{array}{c}\omega_{2}^{\prime} \\
\mathrm{rad} / \mathrm{s}\end{array}$ & $\frac{\omega_{1}}{\omega_{2}}$ \\
\hline - & - $\quad 106$ & - & & - & - & - & 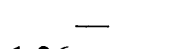 & & - & & - & - & - \\
\hline Fig. $4, n^{0} 2$ & $5 \times 10^{6}$ & 55 & 1,7 & $7 \times 10^{7}$ & $1,1 \times 10^{3}$ & 630 & 1,26 & 2,5 & $\times 10^{5}$ & & $10^{5}$ & 510 & 1,2 \\
\hline Fig. $4, n^{\circ} 3$ & $5 \times 10^{6}$ & 50 & 4 & $\times 10^{7}$ & 500 & 250 & $7,2 \times 10^{-1}$ & & $10^{5}$ & 1,1 & $\times 10^{5}$ & 250 & 1 \\
\hline Fig. $4, n^{0} 4$ & $5 \times 10^{6}$ & 44 & & $10^{8}$ & 220 & 86 & $4,9 \times 10^{-1}$ & 2,2 & $\times 10^{5}$ & 1,5 & $\times 10^{5}$ & 77 & 1,1 \\
\hline Fig. $4, n^{0} 5$ & $5 \times 10^{6}$ & 35 & 2 & $\times 10^{8}$ & 140 & 64 & $1,2 \times 10^{-1}$ & 2,5 & $\times 10^{5}$ & 1,6 & $\times 10^{5}$ & 65 & 1 \\
\hline Fig. $4, n^{\circ} 6$ & $5 \times 10^{6}$ & 29 & 5 & $\times 10^{8}$ & 68 & 31 & $6,3 \times 10^{-2}$ & 1,6 & $\times 10^{5}$ & 1,7 & $\times 10^{5}$ & 25 & 1,3 \\
\hline Fig. $6, n^{0} 5$ & $5 \times 10^{6}$ & 33 & 1,1 & $\times 10^{9}$ & 30 & 12,5 & $\times 10^{-2}$ & 3 & $\times 10^{4}$ & 2 & $\times 10^{5}$ & 9,5 & 1,3 \\
\hline Fig. $6, n^{0} 6$ & $5 \times 10^{6}$ & 26 & 5 & $\times 10^{9}$ & 7 & 2 & $1,3 \times 10^{-2}$ & & $10^{4}$ & 2 & $\times 10^{5}$ & 1 & Z \\
\hline
\end{tabular}

\section{TABLEAU I}

\section{TABLEAU II}

$\begin{array}{ccc}\frac{1}{2 C_{1} \omega} & \omega \mathrm{rad} / \mathrm{s} & R_{\mathrm{s}}(\mathrm{HF}) \Omega \\ - & - & - \\ 1,5 \times 10^{10} & 0,6 & 1,2 \times 10^{10} \\ 1,5 \times 10^{9} & 6,28 & 1,1 \times 10^{9} \\ 1,5 \times 10^{8} & 62,8 & 10^{8} \\ 1,5 \times 10^{7} & 628 & 0,9 \times 10^{7} \\ 1,5 \times 10^{6} & 6280 & 0,8 \times 10^{6}\end{array}$

La figure $8 d$ montre le diagramme asymptotique de $R_{\mathrm{s}}$ et $C_{\mathrm{s}}$; la fréquence de coupure de $C_{\mathrm{s}}$ devrait être déplacée par rapport à la fréquence de coupure de $C_{\mathrm{p}}$ d'un rapport $\left(C_{0} / C_{1}\right)^{1 / 2} \simeq 3 \times 10^{2}$, ce qui est un peu supérieur aux résultats expérimentaux; la fréquence de coupure de $C_{\mathrm{s}}$ correspond bien aussi à $C_{\mathrm{p}}=C_{1}$.

On voit que ce modèle convient assez bien et l'accord avec l'expérience est assez bon. Mais on ne comprend cependant pas pourquoi les pentes de la résistance série en haute fréquence sont voisines de -1 et pourquoi toutes les courbes représentatives de $R_{\mathrm{s}}$ sont confondues.

On a expérimentalement

$$
R_{\mathrm{s}} \simeq R_{0} \frac{1}{1+2 R_{0} C_{1} \omega}
$$

ce qui donnerait en haute fréquence $R_{\mathrm{s}} \simeq \frac{1}{2 C_{1} \omega}$.

Il est toujours possible d'interpréter cet effet par une distribution des temps de relaxation des charges dans le polymère ; et il existe bien une certaine distri- bution, puisqu'il y a des relaxations $\beta$ et $\gamma$ à plus haute fréquence ; mais ces relaxations correspondent à des orientations d'origine dipolaire, donc elles donnent lieu à des dispersions faibles, d'ailleurs sensibles au pourcentage d'eau. La formule précédente peut être expliquée par un processus de diffusion à l'interface entre les couches résistantes et peu résistantes. Supposons que ces dernières se trouvent incluses dans une masse plus résistante : on peut pour tenir compte de la diffusion introduire en série avec $C_{1}$ une capacité de Warburg [17] de valeur

$$
C_{1 \mathrm{w}}=A \omega^{-1 / 2}
$$

où $A$ est une constante.

On aurait alors :

$$
\frac{1}{C_{1}^{\prime} \omega}=\frac{1}{A \omega^{1 / 2}}+\frac{1}{C_{1} \omega} .
$$

Il existerait alors une fréquence de coupure telle que :

$$
A \omega_{\mathrm{W}}^{1 / 2}=C_{1} \omega_{\mathrm{w}} \quad \text { soit } \quad \omega_{\mathrm{w}}=\frac{A^{2}}{C_{1}^{2}}
$$

et aux grandes valeurs de $\omega\left(\omega>\omega_{\mathrm{w}}\right)$

$$
C_{1}^{\prime}=A \omega^{-1 / 2} \text {. }
$$

Remplaçant alors dans $R_{\mathrm{s}}$ on obtiendrait

$$
R_{\mathrm{s}}=R_{0} \frac{1}{1+\omega A^{2} R_{0}^{2}}
$$

et pour rendre compte des résultats expérimentaux :

$$
A^{2} R_{0}=2 C_{1} \text {, c'est-à-dire } A=\left(2 C_{1} / R_{0}\right)^{1 / 2} \text {. }
$$


Généralement une impédance correspondant à ce phénomène de diffusion à l'interface comprend non seulement une capacité, mais également une résistance variable avec la fréquence : il faut admettre que celle-ci est « court-circuitée » par la valeur très faible de $r$.

Dans le cas classique d'une seule interface, l'expression de $A$ est connue. On a alors

$$
A_{0}=\frac{\sqrt{2 D}}{k T} e^{2} c_{0}
$$

où $c_{0}$ est la concentration et $D$ le coefficient de diffusion.

Utilisant la relation d'Einstein

$$
\frac{\mu}{D}=\frac{e}{k T},
$$

et l'expression de la longueur de Debye :

$$
L_{\mathrm{D}}=\left(\frac{\varepsilon k T}{2 c_{0} e^{2}}\right)^{1 / 2}
$$

on peut écrire :

$$
A_{0}=\frac{\sqrt{\varepsilon \sigma}}{L_{\mathrm{D}}}=\sqrt{\frac{C_{0}}{R}}
$$

pour une surface unité.

Dans cette expression $R$ est la résistance de la couche d'épaisseur $L_{\mathrm{D}}$. On peut alors émettre une hypothèse : comparant en effet les deux valeurs de $A$ et $A_{0}$, on voit qu'elles ont même forme, et qu'on peut les relier si on considère dans le diélectrique $n$ couches en série de telle sorte que $C_{1}=C_{0} / n$ et $R_{0}=n R$. Les résultats expérimentaux montreraient que $n=10^{5}$. On aurait également $A_{0}=n A / \sqrt{2}$.

Ce raisonnement permettrait de mieux comprendre l'origine de $C_{1}$ qui n'est pas lié directement à la cristallinité de l'échantillon, mais qui, comme $C_{0}$, est constant, quelle que soit la proportion d'humidité. La constitution du polymère, le nombre des interfaces « internes » et la densité des porteurs ne dépendent pas de cette proportion, et seule varie la mobilité de ces porteurs.
Remplaçant $A$ par sa valeur on a :

$$
\omega_{\mathrm{w}}=\frac{2}{R_{0} C_{1}} .
$$

Aux faibles fréquences $\left(\omega<\omega_{\mathrm{W}}\right)$ la capacité $C_{1}$ devient constante, et les résultats précédents, fréquences de coupure, pentes ne sont pas sensiblement modifiés.

On peut remarquer que l'on pourrait également rendre compte des résultats expérimentaux en utilisant la formule de Goffaux [5] qui tient compte de la variation de l'épaisseur des barrières de potentiel due à la diffusion :

$$
R_{0} C_{1} \omega=\left(\frac{1}{2}\left(\left(1+\omega^{2} \tau_{1 \mathrm{D}}\right)^{1 / 2}-1\right)\right)^{1 / 2}
$$

avec $\tau_{1 \mathrm{D}}=2 R_{0} C_{1}$ car aux fäibles fréquences $C_{1}^{\prime}=C_{1}$.

En résumé, le schéma équivalent donné figure 8 est valable en première approximation, avec des valeurs constantes de ses éléments; il semble mieux décrire les résultats expérimentaux en introduisant une capacité de Warburg : la capacité aux interfaces varierait avec la fréquence par suite d'un processus de diffusion.

Les autres effets de relaxation qui se produisent aux fréquences plus élevées, relaxations $\beta$ et $\gamma$ qui doivent être plus ou moins modifiées par l'introduction d'eau, n'apparaissent pas dans la gamme des fréquences étudiées.

6. Conclusion. - En résumé deux phénomènes de relaxation successifs ont été mis en évidence dans le nylon 66, un phénomène de charge d'espace et un effet de Maxwell-Wagner. On peut relier ces deux effets aux pics $\alpha_{1}$ et $\alpha_{2}$ de thermocourants de dépolarisation [16]. Ces deux effets qui se produisent sur le nylon sec ou humide apparaissent aussi sur un certain nombre de polyamides et polypeptides, par exemple la polyglycine. L'étude comparative de ces relaxations sur divers polymères, d'origine industrielle, ou préparés par polycondensation au laboratoire, doit nous permettre de mieux comprendre la nature de la conduction, tant dans les produits secs que dans les produits hydratés.

\section{Bibliographie}

[1] Starkweather, H. W., Jr, J. Appl. Polymers Science 2 (1959) 129-133.

[2] Gordon, G. A., J. Polymer Science A 2 (1971) 1663-1702.

[3] Seanor, D. A., Electrical Conduction Properties of Polymers, J. Polymer Science C 17 (1967) 195-212.

[4] Hirota, S., Shogo Saito, Tatsuji Nakajima, Kolloid Zeitschrift 213 (1966) 109

[5] Goffaux, R., Mémoire Ac. Royale de Belgique (Sciences) 38 (1968) fasc. 2.

[6] Maitrot, M., Michel, R., Madru, R., Revue Phys. Appl. 6 (1971) 369-376.

[7] Scheiber, D. J., J. Res. Nat. Bur. Stand. 65C (1961) 23.

[8] Martinot, H., Thèse Doctorat, Toulouse, 1963.
[9] Beaumont, J. H. et Jacobs, P. W. M., J. Phys. \& Chem. Solids 28 (1967) 657.

[10] Friauf, R. J., J. Chem. Phys. 22 (1954) 1329.

[11] Raleigh, D. O., J. Phys. \& Chem. Solids 29 (1968) 261

[12] Mac Donald, J. P., Phys. Rev. 92 (1953) 4.

[13] VANDerschueren, H., Bulletin de la Faculté des Sciences Appliquées de l'Université de Liège.

[14] King, G., Medley, J. A., J. Colloid Science 4 (1949) 1.

[15] RosenberG, B., Biopolymer Symposium 1 (1964) 453.

[16] Chatain, D., Lacabanne, C., Maitrot, M., Phys. Stat. Sol. (a) 13 (1972) 303.

[17] Warburg, Ann. Phys. 6 (1901) 125.

[18] Von Hippel, A. R., Dielectric Materials and Applications, (Wiley, New York), 1954. 